\title{
Starspot Activity on Short-period RS CVn Stars
}

\author{
Michael Zeilik \\ Institute for Astrophysics, The University of New Mexico \\ Albuquerque, New Mexico 87131, USA
}

\section{Introduction}

We have yet to understand the magnetic activity cycles of cool close binary systems of sunlike stars. Mutual tidal interactions, as well as magnetic ones, may result from a regime of dynamo models not yet tested, because these have been developed for single stars. To arrive at the basic physics, though, requires that we first examine the phenomenology of magnetic activity for binary systems. In particular, we would like to discover if such activity has a clearly-defined cycle, such as the sun exibits.

Among the proxy indicators of magnetic activity are the $\mathrm{Ca} \mathrm{II} \mathrm{H}$ and $\mathrm{K}$ lines. Strassmeier et al. (1988) used the strength of these lines as the primary criterion for the inclusion of systems in The Catalog of Chromospherically Binary Stars. Of the RS CVn stars in the catalog, 12 have orbital periods of one day or shorter; 9 are eclipsing systems. As part of a decade-long program, we have focussed our observations and models on eight of the short-period group (Hall, 1976): XY UMa, UV Psc, SV Cam, RT And, CG Cyg, ER Vul, BH Vir, and WY Cnc. These close systems are tidally-locked in synchronous rotation and tidally-distorted into Roche lobe configurations.

A few of these systems have archival observations that span decades, hence giving us the long time base to probe for cyclical activity. We can extend these time series with current observations with our CCD-camera at Capilla Peak Observatory and those data collected by other groups. We have completed work on data for SV Cam (Zeilik et al., 1988), RT And (Zeilik et al., 1989), BH Vir (Zeilik et al., 1990a), and WY Cnc (Zeilik et al., 1990b). Most of my comments will relate to these four systems. (We note that these stars are too faint for the current generation of APTs).

\section{Analysis}

Our goal is to apply the simplest possible starspot model (Budding, 1977) to parameterize photometric light curves in a way that reliably and objectively extracts information revelant to photospheric magnetic activity. Using circular, dark spotted regions (Budding and Zeilik, 1987) as the representation of the essential phenomenon, we can optimize for the longitude, latitude, radius, and temperature 
(relative to the photosphere) of the active area. Note we do not believe, in fact, that all regions of activity are circular and without structure. Rather we use this model as an adequate representation to find out information relevant to magnetic activity.

Previous criticisms of photometric modelling (cf. Rodonó, 1986) have aimed at the lack of uniqueness of the starspot solutions. That limitation is especially true when applied to non-eclipsing systems, as has usually been the case so far. Eclipsing systems present a different aspect; the orbital inclinations are known within a degree or so and the eclipses provide fiducial markers for longitude. Hence, eclipsing systems provide a much more constrained modeling problem; the drawback is that we need to separate eclipse and proximity effects to reveal the maculation (distortion) waves. We have done so by modifying Budding's 16-parameter, optimizing curve fitter (Budding, 1973; Budding and Najim, 1980) to generate the model curves for the eclipsing system. These models use Roche lobes for the distorted stars, and include both the reflection and ellipticity effects. The difference between an optimized theoretical curve and the observed one defines the maculation effect. Another curve fitter, corresponding to the case of one or two dark, circular spots, generates an optimized solution to these residuals.

Our programs (Budding and Zeilik, 1987) contain an optimizer routine that uses a variety of methods to search efficiently for a minimum in $\chi^{2}$ hyperspace; the two main ones are a vector search and a parabolic interpolation (Banks, 1989). The $\chi^{2}$ routine contains the particular fitting function appropriate to generate a representation of the eclipsing binary light curve or of the distortion wave from starspots (Budding, 1977). What sets our procedure apart is our information limit analysis, which provides an objective evaluation of the results from the optimized parameters, in the sense of the information content of the data.

As detailed in Budding and Najim (1980), our programs evaluate the curvature Hessian in the vicinity of the optimal solution in $\chi^{2}$ hyperspace. We also calculate the error matrix, which is the inverse of the curvature Hessian. For mathematicallydeterminate solutions, the curvature Hessian will have a positive eigenvalue for each optimized parameter. We approach the information limit by increasing the number of parameters until the Hessian becomes nonpositive definite; we then know that we have exceeded the information inherent in the data.

\section{Results for the short-period systems}

Our main results to date, which apply to the primary (hotter) star in these shortperiod systems, are: (1) The primary activity signature is two active longitude belts (ALBs) at the quadrature points; one belt is more active than the other at any given time. (2) The secondary activity signature is that the active regions appear most often at high latitudes (near $45^{\circ}$ ). (3) The spotted regions cover large areas of the primary, active stars (radii are typically $10^{\circ}$ ). (4) These cool areas have temperatures about $1500 \mathrm{~K}$ below that of the photosphere with a variation of 
only a few hundred Kelvins (for those light curves for which we have simultaneous $V$ and $R$ or $I$ data).

A few comments about specific systems. SV Cam: over the time from 1928 to 1984 , the ALB around $270^{\circ}$ has been more active. The mean latitude value for determinate solutions is $58^{\circ} 4$. The differential rotation rates within the ALBs are much smaller than the sun's. RT And: again, the ALB near $270^{\circ}$ seems the most active (from 1920 to 1989 ). The mean value of determinate latitudes was $53^{\circ}$. The orbit does have a small eccentricity $(\mathrm{e} \sim 0.03)$. BH Vir: both ALBs have roughly the same activity from 1953-1986. The mean value of the determinate latitudes was $31^{\circ}$. The temperature contrast averaged only $1100 \mathrm{~K}$. WY Cnc: The ALB at $90^{\circ}$ seems more active in 1964-1989. The mean latitude of determinant's solutions was $15: 8$, this is the only system that we have analyzed that resulted in low-latitude spots. The temperature contrast was about $1500 \mathrm{~K}$.

We note that the ALB activity appears binary. When one is "on", the other is "off", at least in terms of large spotted regions. (We cannot "see" the spots if their radii is less than $5^{\circ}$ ). We have so far found no instances in which we have a clear indication of both ALBs containing activity at the same time. Hence, we may have a magnetic resonance occurring in the longitudinal distribution of the magnetic fields.

This research was supported in art by NSF Grant AST-8903174.

\section{References}

Budding, E.: 1973, Ap. Space Sci. 22, 87

Budding, E.: 1977, Ap. Space Sci. 48, 287

Budding, E., Najim, N.N.: 1980, Ap. Space Sci. 72, 369

Budding, E., Zeilik, M.: 1987, Astrophys. J. 319, 827

Nelson, E., Zeilik, M: 1990, Astrophys. J. 349, 163

Rodonó, M.: 1986, in Cool stars, Stellar Systems, and the Sun, ed. M. Zeilik and D. Gibson (Heidelberg: Springer-Verlag), p. 470

Strassmeier, K.G., Hall, D.S., Zeilik, M., Nelson, E., Eker, Z., Fekel, F.C.: 1988, Astrophys. Astron. Suppl. 72, 291

Vogt, S.S., Penrod, G.D.: 1983, Pub. Astron. Soc. Pacific 95, 65

Zeilik, M. De Blasi, C., Rhodes, M., Budding, E.: 1988, Astrophys: J. 332, 293

Zeilik, M., Cox, D.A., De Blasi, C., Phodes, M., Budding, E.: 1989, Astrophys. J. 345, 991

Zeilik, M., Ledlow, M., Phodes, M., Arevalo, M.J., Budding, E.: 1990a, Astrophys. J. $\mathbf{3 4 5}, 352$

Zeilik, M., Cox, D.A., Ledlow, M.J., Phodes, M., Heckert, P.A., Budding, E.: 1990b, Astrophys. J. 363, in press 